\title{
Optimal Caching in Multicast 5G Networks with Opportunistic Spectrum Access
}

\author{
Mostafa Emara*, Hesham ElSawy ${ }^{\dagger}$, Sameh Sorour $^{\ddagger}$, Samir Al-Ghadhban*, Mohamed-Slim Alouini ${ }^{\dagger}$ and Tareq Y. Al-Naffouri $^{\dagger}$ \\ *King Fahd University of Petroleum and Minerals (KFUPM), Saudi Arabia \\ ${ }^{\dagger}$ King Abdullah University of Science and Technology (KAUST), Saudi Arabia \\ ${ }^{\ddagger}$ University of Idaho, USA
}

\begin{abstract}
Cache-enabled small base station (SBS) densification is foreseen as a key component of $5 \mathrm{G}$ cellular networks. This architecture enables storing popular files at the network edge (i.e., SBS caches), which empowers local communication and alleviates traffic congestions at the core/backhaul network. This paper develops a mathematical framework, based on stochastic geometry, to characterize the hit probability of a cache-enabled multicast 5G network with SBS multi-channel capabilities and opportunistic spectrum access. To this end, we first derive the hit probability by characterizing opportunistic spectrum access success probabilities, service distance distributions, and coverage probabilities. The optimal caching distribution to maximize the hit probability is then computed. The performance and trade-offs of the derived optimal caching distributions are then assessed and compared with two widely employed caching distribution schemes, namely uniform and Zipf caching, through numerical results and extensive simulations. It is shown that the Zipf caching almost optimal only in scenarios with large number of available channels and large cache sizes.
\end{abstract}

Keywords-Caching System; Stochastic Geometry; Opportunistic System Access.

\section{INTRODUCTION}

The rapid proliferation of social networking along with the advancement in smart devices impose increasing traffic burden on cellular operators. Such evolution entails massive numbers of connections with several data hungry application. For instance, video streaming is a main contributor to the traffic burden which is anticipated to consume around $82 \%$ of the total internet traffic by 2020 [1]. Fortunately, with the aid of data analytics on social networks, operators can estimate the popularity of contents (e.g., videos) in terms of the volume of user demand. Relying on this fact, the fifth generation $(5 \mathrm{G})$ of cellular networks is evolving towards datacentric networking. In particular, cellular operators will i) densify their networks via cache enabled small base stations (SBSs); ii) implement proactive cacheing for popular content at the network edge; and iii) utilize multicasting for common demands [2]. This clearly improves the spatial frequency reuse, relieves the backhaul from congestion, and better utilizes the radio resources.

A fundamental and widely employed design objective in data-centric networks is to maximize the probability of users finding requested popular files in the cache of an accessible edge SBS, denoted as the hit probability. More precisely, the hit probability is defined by the joint event of finding an SBS that i) is caching the requested popular file; and ii) is capable of serving the requesting user with at least a minimum pre-defined threshold of signal-to-interference-plusnoise-ratio (SINR). Maximizing the hit probability requires optimal content placement in the SBSs along with spectrum and interference management, since the catering SBS is not necessarily the geographically closest SBS to the requesting user. However, the literature mostly focuses on the optimal caching problem and overlooks the appropriate interference aspects of cellular networks. For instance, the authors in [3] propose an optimal caching distribution that maximizes the hit probability in order to alleviate core network delay and achieve minimal file downloading time. In [4], a joint routing and caching problem is formulated to maximize the content requests served locally by SBSs. However, the work in [3], [4] assumes interference free environment. Using stochastic geometry, the interference effect is accounted for in [5]-[7], where optimal caching distribution is proposed to maximize the hit probability. However, the work in [5]-[7] considers a single channel system in a fully loaded SBS scenario, which contradicts with the typical multi-channel capabilities of cellular networks and thus leads to a pessimistic performance assessment. A joint optimal file placement and multicasting in a multi-channel system is proposed in [8]. However, [8] follows a rigid frequency reuse scheme, which is well-known to underutilize the spectrum resources [9]. In [10], we analyzed the performance of cache-enabled networks with opportunistic spectrum access in a multi-channel environment. However, [10] considers a single-user unicast scenario.

This paper develops a mathematical model, based on the stochastic geometry, for cache-enabled 5G networks with opportunistic spectrum access (OSA) in a multi-channel environment. We assume a Poisson point process (PPP) cellular networks with Zipf distributed file popularity. In contrast to the literature, we consider both caching design and OSA based interference management for multicast content delivery. Since the requested file may not be served from the geographically nearest SBS, the catering SBS utilize OSA to alleviate interfering with all SBSs that are closer to the demanding user. To this end, we formulate an optimization problem for maximizing the hit probability, in which the optimal cache design is obtained and compared to conventional Zipf and uniform based caching. The main contributions of this paper are as follows: 
- OSA: Exploiting the available multi-channels that are universally reused by all SBSs, the catering SBS opportunistically transmits the requested file on a channel that is not used by any closer SBS to the requesting user. This would increase the hit probability in case the requested file is not cached in a closer SBS.

- Multicasting: The SBSs adopt multicasting to better utilize the available spectrum by reducing the number of channel used by each BS. This would create more spectrum opportunities for OSA and diversify interference over the available channels.

- Optimal Caching: In contrast to the popularity based caching that is agnostic to the network parameters, optimal caching adapts to the network conditions to maximize the hit probability.

- Mathematical Model: To the best of the authors knowledge, this paper presents the first stochastic geometry based mathematical model for a multicast OSA cache enabled network in a multi-channel environment. The developed mathematical model is verified via independent Monte Carlo simulations.

- Design Insights: The paper reveals novel insights to the optimal caching strategy. For instance, storing the most popular files only at all SBSs is the optimal caching strategy in interference aggressive scenario. When the interference effect is relieved (e.g., by increasing the number of channels or decreasing the SINR threshold) diversifying the stored files over the SBSs with a certain scenario-dependent storage probabilities becomes the optimal strategy.

\section{SySTEM Model}

Network Model: We assume that the SBSs and the users are distributed in $\mathbb{R}^{2}$ according to two independent homogenous PPPs, $\boldsymbol{\Phi}_{b}$ and $\boldsymbol{\Phi}_{u}$, with intensities $\lambda_{b}$ and $\lambda_{u}$, respectively. In the considered downlink transmissions, all SBSs transmit with the same power $P$ and share a common set of channels $\mathbf{S}$ with cardinality $|\mathbf{S}|$. The signal attenuation due to propagation is characterized by the power-law distance depended path-loss model $r^{-\eta}$, where $r$ is the propagation distance and $\eta>2$ is an environment dependent exponent. A Rayleigh fading environment is assumed with independent and identically distributed channel gains with unit mean powers. According to Slivnyak-Mecke theorem [11], there is no loss in generality to focus on a test user located at the origin (i.e., typical user), to analyze the network performance.

Caching Model: We consider a finite library of popular files, denoted as $\mathcal{J}=\left\{c_{1}, c_{2}, \ldots, c_{J}\right\}$. It is assumed that all files have the same length. However, this analysis may be still applied with files of different sizes by chopping each file into equal length packets. The files' popularity follows a Zipf distribution that is known a priori for the network operator. The popularity distribution is expressed as:

$$
a_{j}=\frac{j^{-\gamma}}{\sum_{j=1}^{J} i^{-\gamma}}
$$

where $a_{j}$ is the probability that a generic user requests the file $c_{j}$, and $\gamma$ is the Zipf parameter that governs the popularity distribution skewness. Larger (smaller) $\gamma$ increases (decreases) the discrepancies among the files popularity and implies that fewer (more) files are frequently requested. Without loss of generality (WLOG), it is assumed that the files of the library are ordered according to their popularity, i.e., $a_{1} \geq a_{2} \geq \cdots \geq$ $a_{J}$. Each SBS has a cache memory of size $M<J$ files. It is assumed that each SBS independently chooses a combination $x \in \mathcal{X}$ of different ${ }^{1} M$ files to store in its cache, where $\mathcal{X}=$ $\{1,2, \ldots, X\}$ denotes the set of all possible combinations with a set cardinality $X=\left(\begin{array}{c}J \\ M\end{array}\right)$. Let $p_{x}$ denotes the probability that a generic SBS stores a combination $x \in \mathcal{X}$. Then, $p_{x}$ satisfies the following constraints

$$
\begin{aligned}
& 0 \leq p_{x} \leq 1 \quad, x=1,2, \ldots, X \\
& \sum_{x=1}^{X} p_{x}=1
\end{aligned}
$$

Thus the probability that a generic SBS stores a particular file $c_{j}$ is given by

$$
b_{j}=\sum_{x \in \mathcal{X}_{j}} p_{x}
$$

where $\mathcal{X}_{j} \subseteq \mathcal{X}$ constitutes all the combinations that have the file $c_{j}$. The caching distribution $\mathbf{P}=\left\{p_{x}: x \in \mathcal{X}\right\}$ is considered as a key design parameter that controls the network performance as explained in the sequel.

OSA: WLOG, it is assumed that the indices of the SBSs are ordered according to their distances from the test user at the origin. Each user requesting file $c_{j}$ is associated to the nearest SBS that caches a combination $x \in \mathcal{X}_{j}$ that includes the requested file $c_{j}$. Since $M<J$, the catering $\mathrm{BS}$ is not necessarily the geographically closest SBS. We denote the catering SBS with its distance order from the requesting user. When the user is served by the $n^{\text {th }}$ SBS, the requested file is transmitted over a channel that is not used by the $(n-1)$ closer SBSs if any still exists. Otherwise, the serving SBS randomly and uniformly selects a channel to transmit the requested file. Information about the channels used by the $(n-1)$ closer SBSs to the demanding user can be estimated via spectrum sensing by the user or through signaling among the SBSs.

Multicasting: All users that request the same file from an SBS are scheduled on the same frequency channel in a multicast fashion. It is assumed that the time is divided into slots and a file transmission consumes a single time slot. For synchronized transmission, requested file delivery within a time slot is delayed to the start of the next slot. Multicasting implies that each SBS uses at most $M$ channels

\footnotetext{
${ }^{1}$ File redundancy at the same cache is avoided as it wastes the memory resources at no additional benefit.
} 
to serve its requesting users if all its cached files are requested.

Performance Analysis: The hit probability, characterizing the joint event of caching the file at the network edge and successfully transmitting the file with SINR greater than a certain threshold $\beta$, is the main performance metric considered in this paper. According to the law of total probability, the hit probability can be expressed as:

$$
\mathcal{H}=\sum_{j=1}^{J} a_{j} \sum_{n=1}^{\infty} b_{j}\left(1-b_{j}\right)^{(n-1)}\left(\mathcal{C} \mathcal{O}_{n}+\mathcal{C}^{\prime}\left(1-\mathcal{O}_{n}\right)\right),
$$

where $a_{j}$ is the probability that the typical user requests file $c_{j} ; b_{j}\left(1-b_{j}\right)^{n-1}$ is the probability that the desired file is not stored in any of the $(n-1)$ closer SBSs and is available at the $n^{\text {th }} \mathrm{SBS} ; \mathcal{C}$ denotes the coverage probability when OSA is successful, $\mathcal{C}^{\prime}$ denotes the coverage probability when the OSA fails; and $\mathcal{O}_{n}$ represents the OSA success probability when the requesting user is served by the $n^{t h} \mathrm{SBS}$.

\section{OSA SuCCESS PRobAbility}

The OSA process is said to be successful when the catering SBS of order $n$ finds a free channel not used by any of the $(n-1)$ closer SBSs to the requesting user. $\mathcal{O}_{1}$ is trivially one as the catering SBS is the geographically closest one. Otherwise, the first building block to compute $\mathcal{O}_{n}$ for $n>1$ is the probability that each file $c_{j}$ is requested from a generic SBS, which is given by

$$
\zeta_{j}=\mathbb{P}\left[\mathcal{U}_{j} \geq 1\right]=1-\left[\mathrm{v}^{-1} \frac{a_{j} \lambda_{u}}{b_{j} \lambda_{b}}+1\right]^{-\mathrm{v}},
$$

where $\mathrm{v}=3.575$ is a constant related to the PPP Voronoi cell area distribution. Exploiting the fact that each file is transmitted on a unique channel, the probability mass function (PMF) of the channels used be a generic SBS is given by the following lemma

Lemma 1: Let $r_{n}$ be the distance between the catering SBS and the requesting user, then the PMF of the number of used channels by a generic SBS inside $r_{n}$, denoted as $\mathcal{N}_{c,-j}$, is given by

$$
\begin{aligned}
\mathbb{P}\left[\mathcal{N}_{c,-j}=\kappa\right]= & \sum_{x \in \mathcal{X}_{-j}} \frac{p_{x}}{1-b_{j}} \sum_{\{\mathcal{A} \subseteq x:|\mathcal{A}|=\kappa\}}\left[\prod_{c_{m} \in \mathcal{A}} \zeta_{m}\right. \\
& \left.\cdot \prod_{c_{m} \in x \backslash \mathcal{A}}\left(1-\zeta_{m}\right)\right], 0 \leq \kappa \leq M
\end{aligned}
$$

where the subscript $(-j)$ indicates the fact that the requested file $c_{j}$ is not stored by any of the SBSs within $r_{n}$.

Proof: Please refer to Appendix A

This OSA process imposes scheduling interactions among the $(n-1)$ nearer SBSs, which complicates the analysis. For tractability, the OSA scheduling interactions among the $(n-1)$ closer SBSs is ignored, in which the accuracy of this approximation is validated in Section-VI. Exploiting this approximation and following [12], the PMF $\mathcal{P}_{n-1}(\kappa)$ can be expressed as:

$$
\begin{gathered}
\mathcal{P}_{n-1}(\kappa) \approx \sum_{v=0}^{\min (\kappa,(n-2) M)} \mathcal{P}_{n-2}(v) \sum_{w=\kappa-v}^{\kappa}\left[\mathbb{P}\left[\mathcal{N}_{c,-j}=w\right]\right. \\
\left.\cdot\left(\begin{array}{c}
w \\
w-(\kappa-v)
\end{array}\right)\left(\frac{v}{|\mathbf{S}|}\right)^{w-(\kappa-v)}\left(1-\frac{v}{|\mathbf{S}|}\right)^{\kappa-v} \mathbb{1}_{\{w \leq M\}}\right] \\
0 \leq \kappa \leq \min (|\mathbf{S}|, M(n-1))
\end{gathered}
$$

where $\mathbb{1}_{\{\cdot\}}$ is the indicator function. Using (5)-(7), the OSA success probability can be expressed as

$$
\mathcal{O}_{n}=\sum_{\kappa=0}^{|\mathbf{S}|-1} \mathcal{P}_{n-1}(\kappa)=1-\mathcal{P}_{n-1}(|\mathbf{S}|) .
$$

\section{File transmission SUCCESS PRobability}

This section characterizes the SINR distribution, when the OSA succeeds and fails, to find the file transmission success probability.

\section{A. Distance distribution}

The first step to analyze the SINR, is to characterize the service distance distribution. Conditioning that the catering SBS is of order $n$, the probability density function (PDF) of the serving distance $r_{n}$ is given by [13, Lemma 3]

$$
f_{r_{n}}(r)=\frac{2\left(\pi \lambda_{b} r^{2}\right)^{n}}{r \Gamma(n)} e^{-\pi \lambda_{b} r^{2}}, 0 \leq r \leq \infty
$$

According to the PPP, conditioning on $r_{n}$, the (n-1) nearer SBS are uniformly and independently scattered in a disc of radius $r_{n}$ centered at the origin, denoted as $\mathcal{D}_{\circ}\left(r_{n}\right)$. Hence, the distance distribution between the user and a randomly selected SBS of the (n-1) nearer SBS is given by

$$
f_{r_{i}}\left(x \mid r_{n}\right)=\frac{2 x}{r_{n}^{2}} \quad, 0 \leq x \leq r_{n} .
$$

The distance distribution in (10) is needed to characterize interference when the OSA fails, i.e., when the test user experience interference from $1 \leq \kappa \leq(n-1)$ SBSs within $r_{n}$.

\section{B. SINR analysis}

The coverage probability, is defined as the probability that the transmitted file is received at the test user with SINR greater than the detection threshold $\beta$. Note that the SINR distribution will highly depend on whether the OSA succeeded or not. In case the OSA succeeds, the test user does not receive interference from the closer $(n-1)$ SBSs and the coverage probability is defined as

$$
\begin{aligned}
\mathcal{C} & =\mathbb{P}\left[\frac{P h_{n} r_{n}^{-\eta}}{\sigma_{n}^{2}+\mathcal{I}_{\text {out }}} \geq \beta\right] \\
& =\mathbb{P}\left[\frac{P h_{n} r_{n}^{-\eta}}{\sigma_{n}^{2}+\sum_{y_{i} \in \tilde{\mathbf{\Phi}}_{b} \backslash \mathcal{D}_{\circ}\left(r_{n}\right)} P h_{i}\left\|y_{i}\right\|^{-\eta}} \geq \beta\right]
\end{aligned}
$$

where $h_{n}\left(\right.$ resp. $\left.h_{i}\right)$ is the channel gain between the typical user and its catering SBS (resp. the $i^{\text {th }}$ interfering SBS), $\|\cdot\|$ is the Euclidean norm, $\tilde{\boldsymbol{\Phi}}_{b} \subset \boldsymbol{\Phi}_{b}$ is the set of interfering 
SBSs that are using the same channel allocated to the test user, and $\sigma_{n}^{2}$ is noise variance. Due to multicasting, multichannel, and the probabilistic file demand, not all SBSs are using all channels and the interference is diversified over available channels [12]. Ignoring the OSA interactions among the interfering SBSs, the probability that a generic SBS is using the same channel as the test user is characterized in the following lemma

Lemma 2: Let $\mathcal{T}$ be the probability that a generic SBS outside $r_{n}$ is using a randomly and uniformly selected channel out of $|\mathbf{S}|$ available channels:

$$
\mathcal{T}= \begin{cases}\sum_{n=0}^{M} \mathbb{P}\left[\mathcal{N}_{c}=n\right] \frac{n}{|\mathbf{S}|}, & \text { if } M<|\mathbf{S}| . \\ 1-\sum_{n=0}^{|\mathbf{S}|-1} \mathbb{P}\left[\mathcal{N}_{c}=n\right] \frac{|\mathbf{S}|-n}{|\mathbf{S}|}, & \text { if } M \geq|\mathbf{S}| .\end{cases}
$$

where $\mathbb{P}\left[\mathcal{N}_{c}=\kappa\right]$ can be given by:

$$
\mathbb{P}\left[\mathcal{N}_{c}=\kappa\right]=\sum_{x \in \mathcal{X}} p_{x} \sum_{\{\mathcal{A} \subseteq x:|\mathcal{A}|=\kappa\}} \prod_{c_{m} \in \mathcal{A}} \zeta_{m} \prod_{\substack{c_{m} \in x \backslash \mathcal{A} \\, 0 \leq \kappa \leq M}}\left(1-\zeta_{m}\right)
$$

Proof: Please refer to Appendix B

Note that $\mathbb{P}\left[\mathcal{N}_{c}=\kappa\right]$ in (13) is the PMF of used number channels by a generic SBS outside $\mathcal{D}_{\circ}\left(r_{n}\right)$. It can be obtained using the same procedure as in Lemma 1 without any restriction on the combination that can be stored by the SBS.

In case of OSA failure, there are interfering SBSs within $r_{n}$. Consequently, the coverage probability becomes

$$
\mathcal{C}^{\prime}=\mathbb{P}\left[\frac{P h_{n} r_{n}^{-\eta}}{\sigma_{n}^{2}+\mathcal{I}_{\text {in }}+\mathcal{I}_{\text {out }}} \geq \beta\right]
$$

where $\mathcal{I}_{\text {out }}$ is given in (11) and $\mathcal{I}_{\text {in }}=$ $\sum_{y_{i} \in \tilde{\boldsymbol{\Phi}}_{b} \cap \mathcal{D}_{\circ}\left(r_{n}\right)} P h_{i}\left\|y_{i}\right\|^{-\eta}$. Note that not all the SBSs in $\tilde{\boldsymbol{\Phi}}_{b} \cap \mathcal{D}_{\circ}\left(r_{n}\right)$. The probability that a SBS in $\tilde{\boldsymbol{\Phi}}_{b} \cap \mathcal{D}_{\circ}\left(r_{n}\right)$ is using the same channel as the test user, denoted by $\mathcal{T}_{-j}$, is given by (12) with $\mathcal{N}_{c,-j}$ substitutes $\mathcal{N}_{c}$. Note that $\mathcal{T}_{-j}$ is different than $\mathcal{T}$ due to the fact that the requested file $c_{j}$ is not catered by any of the SBSs within $\mathcal{D}_{\circ}\left(r_{n}\right)$.

Conditioning on the distance $r_{n}$, the conditional coverage probabilities are given by

$$
\begin{aligned}
\mathcal{C}\left(r_{n}\right) & =e^{\frac{-\beta r_{n}^{\eta} \sigma_{n}^{2}}{P}} \mathcal{L}_{\mathcal{I}_{\text {out }}}\left(\frac{\beta r_{n}^{\eta}}{P}\right) \\
\mathcal{C}^{\prime}\left(r_{n}\right) & =e^{\frac{-\beta r_{n}^{\eta} \sigma_{n}^{2}}{P}} \mathcal{L}_{\mathcal{I}_{\text {out }}}\left(\frac{\beta r_{n}^{\eta}}{P}\right) \mathcal{L}_{\mathcal{I}_{\text {in }}}\left(\frac{\beta r_{n}^{\eta}}{P}\right)
\end{aligned}
$$

where $\mathcal{L}_{\mathcal{I}}(t)=\mathbb{E}\left[e^{-t \mathcal{I}}\right]$ denotes the Laplace transform (LT) of $\mathcal{I}$. $\mathcal{L}_{\mathcal{I}_{\text {in }}}$ and $\mathcal{L}_{\mathcal{I}_{\text {out }}}$ are the Laplace transforms (LTs) of $\mathcal{I}_{\text {in }}$ and $\mathcal{I}_{\text {out }}$, respectively. Ignoring the OSA interactions between the SBSs and assuming that each SBS randomly and uniformly selects the channels for catering the requested files, the LTs of the aggregate interference from SBSs outside and inside $\mathcal{D}_{\circ}\left(r_{n}\right)$ can be obtained as

$\mathcal{L}_{\mathcal{I}_{\text {out }}}\left(\frac{\beta r_{n}^{\eta}}{P}\right)=\exp \left(-\frac{2 \pi \mathcal{T} \lambda_{b} \beta r_{n}^{2}}{\eta-2}{ }_{2} F_{1}\left(1,1-\frac{2}{\eta}, 2-\frac{2}{\eta},-\beta\right)\right)$ and

$$
\begin{aligned}
& \mathcal{L}_{\mathcal{I}_{i n}}\left(\frac{\beta r_{n}^{\eta}}{P}\right)=\left(1-\mathcal{T}_{-j}+2 \mathcal{T}_{-j} \vartheta(\beta, \eta)\right)^{n-1}-\left(1-\mathcal{T}_{-j}\right)^{n-1} \\
& \text { where } \quad \vartheta(\beta, \eta)=\beta^{\frac{2}{\eta}} \int_{z=0}^{\beta^{-\frac{1}{\eta}}} \frac{z}{1+z^{-\eta}} d z
\end{aligned}
$$

Note that (16) and (17) follow by approximating the interfering SBSs outside and inside $\mathcal{D}_{\circ}\left(r_{n}\right)$ by independent PPPs with intensities $\mathcal{T} \lambda_{b}$ and $\mathcal{T}_{-j} \lambda_{b}$, respectively. Hence, the same methodologies in [10, Lemma 1$]$ and [14, Appendix A] can be used to obtain (16) and (17), respectively. Averaging over the PDF of the serving distance $r_{n}$ givin in (9), the unconditional coverage probabilities are given by (18).

In the special case of interference-limited networks (i.e., $\sigma_{n}^{2}=0$ ) and path loss exponent $\eta=4$ (which is common for urban outdoor environments), the coverage probabilities in (18) simplifies to the closed-form expressions given by

$$
\begin{aligned}
\mathcal{C}= & (1+\mathcal{T} \sqrt{\beta} \arctan (\sqrt{\beta}))^{-n} \\
\mathcal{C}^{\prime}= & {\left[\left(1-\mathcal{T}_{-j} \sqrt{\beta} \arctan \left(\frac{1}{\sqrt{\beta}}\right)\right)^{n-1}-\left(1-\mathcal{T}_{-j}\right)^{n-1}\right] } \\
& \cdot(1+\mathcal{T} \sqrt{\beta} \arctan (\sqrt{\beta}))^{-n}
\end{aligned}
$$

Finally, by substituting (18) and (19) into (4), we obtain, respectively, the general and closed-form expressions of the hit probability for the proposed system. The hit probability expression reveals its dependency on both the network parameters ( $\lambda_{b}, \lambda_{u}$, and the SNR $\left.\frac{P}{\sigma^{2}}\right)$ and the caching parameters $\left(a_{j}, b_{j}\right.$, and $\left.p_{x}\right)$.

\section{Optimal CACHing Distribution}

This section derives the optimal caching distribution $\mathbf{P}^{*}=$ $\left\{p_{x}{ }^{*}, x \in \mathcal{X}\right\}$ that maximizes the hit probability, which is obtained via solving the following formulation

$$
\begin{array}{cl}
\max _{\mathbf{P}} & \mathcal{H}\left(p_{1}, p_{2}, \ldots, p_{x}\right) \\
\text { subject to } & 0 \leq p_{x} \leq 1 \quad, x=1,2, \ldots, X \\
& \sum_{x=1}^{X} p_{x}=1
\end{array}
$$

As the hit probability depends on the caching distribution $p_{x}, x \in \mathcal{X}$ (captured by $b_{j}, \mathcal{T}, \mathcal{T}_{-j}$, and $\mathcal{O}_{n}$ ) in a complex manner, it is hard to solve (20). Hence, we consider the asymptotic scenario by assuming that the network is interferencelimited (i.e., the interference dominates the noise power, thus SNR $\left.\frac{P}{\sigma^{2}} \rightarrow \infty\right)$ and heavy loaded (i.e, $\lambda_{u} \rightarrow \infty$ ). Under this scenario, $\mathbb{P}\left[\mathcal{N}_{c,-j}=\kappa\right]$ and $\mathbb{P}\left[\mathcal{N}_{c}=\kappa\right]$ in (5) and (13) simplifies to

$$
\left.\mathbb{P}\left[\mathcal{N}_{c,-j}=\kappa\right]\right|_{\infty}=\left.\mathbb{P}\left[\mathcal{N}_{c}=\kappa\right]\right|_{\infty}=\left\{\begin{array}{ll}
1 & , \kappa=M \\
0 & , \text { otherwise }
\end{array} .\right.
$$

Consequently, the thinning factors $\mathcal{T}$ and $\mathcal{T}_{-j}$ reduce to

$$
\mathcal{T}_{\infty}=\mathcal{T}_{-j, \infty}= \begin{cases}\frac{M}{|\mathbf{S}|} & , M<|\mathbf{S}| \\ 1 & , M \geq|\mathbf{S}|\end{cases}
$$




$$
\begin{aligned}
\mathcal{C} & =\frac{2\left(\pi \lambda_{b}\right)^{n}}{\Gamma(n)} \int_{0}^{\infty} v^{2 n-1} e^{\frac{-\beta v^{\eta} \sigma_{n}^{2}}{P}} \exp \left(-\frac{2 \pi \mathcal{T} \lambda_{b} \beta v^{2}}{\eta-2}{ }_{2} F_{1}\left(1,1-\frac{2}{\eta}, 2-\frac{2}{\eta},-\beta\right)\right) e^{-\pi \lambda_{b} v^{2}} d v \\
\mathcal{C}^{\prime} & =\mathcal{C}\left[\left(1-\mathcal{T}_{-j}+2 \mathcal{T}_{-j} \vartheta(\beta, \eta)\right)^{n-1}-\left(1-\mathcal{T}_{-j}\right)^{n-1}\right]
\end{aligned}
$$

With the SNR $\frac{P}{\sigma^{2}} \rightarrow \infty$, the coverage probability $\mathcal{C}$ in (18) reduces to

$$
\begin{aligned}
\mathcal{C}_{\infty}= & \frac{2\left(\pi \lambda_{b}\right)^{n}}{\Gamma(n)} \int_{0}^{\infty} v^{2 n-1} \exp \left(-\pi \lambda_{b}\left[1+\frac{2 \beta \mathcal{T}_{\infty}}{\eta-2}\right.\right. \\
& \left.\left.\cdot{ }_{2} F_{1}\left(1,1-\frac{2}{\eta}, 2-\frac{2}{\eta},-\beta\right)\right] v^{2}\right) d v .
\end{aligned}
$$

The integral $\int_{0}^{\infty} v^{a} e^{-\alpha v^{2}} d v=\frac{\Gamma(\delta)}{2 \alpha^{\delta}}, \operatorname{Re}(\alpha)>0, \operatorname{Re}(a)>0$ and $\delta=\frac{a+1}{2}$ [15]. Thus, $\mathcal{C}_{\infty}$ can be expressed as

$$
\mathcal{C}_{\infty}=\left(1+\frac{2 \beta \mathcal{T}_{\infty}}{\eta-2}{ }_{2} F_{1}\left(1,1-\frac{2}{\eta}, 2-\frac{2}{\eta},-\beta\right)\right)^{-n} .
$$

Given that each SBS uses $M$ channels when $\lambda_{u} \rightarrow \infty$, the OSA success probability $\left.\mathcal{O}_{n}\right|_{\infty}$ can be given by

$$
\left.\mathcal{O}_{n}\right|_{\infty}=\left.\sum_{\kappa=0}^{|\mathbf{S}|-1} \mathcal{P}_{n-1}\right|_{\infty}(\kappa)=1-\left.\mathcal{P}_{n-1}\right|_{\infty}(|\mathbf{S}|),
$$

where the PMF $\left.\mathcal{P}_{n-1}\right|_{\infty}(\kappa)$ is given by Eq. (26). Therefore, the asymptotic hit probability $\mathcal{H}_{\infty}$ is given by

$$
\mathcal{H}_{\infty}=\sum_{j=1}^{J} a_{j} \sum_{n=1}^{\infty} b_{j}\left(1-b_{j}\right)^{(n-1)} \varphi(n, M,|\mathbf{S}|),
$$

where $\varphi(n, M,|\mathbf{S}|)=\left.\mathcal{C}_{\infty} \mathcal{O}_{n}\right|_{\infty}+\mathcal{C}_{\infty}^{\prime}\left(1-\left.\mathcal{O}_{n}\right|_{\infty}\right)$

The asymptotic hit probability is a function of $p_{x}$ through $b_{j}$ via the linear relation between $b_{j}$ and $p_{x}$ in (3). Thus, the optimization problem in (20) is equivalent to [16, Lemma 2]

$$
\begin{aligned}
\mathcal{H}_{\infty}^{*}=\max _{\mathbf{b}} & \sum_{j=1}^{J} a_{j} \sum_{n=1}^{\infty} b_{j}\left(1-b_{j}\right)^{(n-1)} \varphi(n, M,|\mathbf{S}|) \\
\text { subject to } & 0 \leq b_{j} \leq 1 \quad, j=1,2, \ldots, J \\
& \sum_{j=1}^{J} b_{j}=M .
\end{aligned}
$$

It is easy to show the concavity of the objective function $\mathcal{H}_{\infty}$ by confirming that the first derivative is $\geq 0$ and the second derivative is $\leq 0$. Also, the constraints are linear, which implies that the necessity and sufficiency conditions for optimality exist. The KKT Lagrangian function of the asymptotic problem is given by:

$$
\begin{aligned}
L(\mathbf{b}, \mathbf{w}, \mu, v) & =\sum_{j=1}^{J} a_{j} \sum_{n=1}^{\infty} b_{j}\left(1-b_{j}\right)^{(n-1)} \varphi(n, M,|\mathbf{S}|) \\
& +v\left(M-\sum_{j=1}^{J} b_{j}\right)+\sum_{j=1}^{J} w_{j}\left(b_{j}-1\right)-\sum_{j=1}^{J} \mu_{j} b_{j} .
\end{aligned}
$$

For brevity, we omit the details of finding the optimal file placement $b_{j}^{*}$. Thus, $b_{j}^{*}$ is given by

$$
b_{j}^{*}=\left\{\begin{array}{ll}
0 & , v^{*}<a_{j}(\varphi(1, M,|\mathbf{S}|)-\varphi(2, M,|\mathbf{S}|)) \\
1 & , v^{*}>a_{j} \sum_{n=1}^{\infty} \varphi(n, M,|\mathbf{S}|) \\
\xi\left(v^{*}\right) & , \text { otherwise }
\end{array},\right.
$$

where $\xi\left(v^{*}\right)$ is the solution of $v^{*}=a_{j}[\varphi(1, M,|\mathbf{S}|)+$ $\left.\sum_{n=2}^{\infty}\left(1-n b_{j}^{*}\right)\left(1-b_{j}^{*}\right)^{n-2} \varphi(n, M,|\mathbf{S}|)\right]^{2}$ that satisfies $\sum_{j=1}^{J} b_{j}=M$.

Finally, the optimal caching $\mathbf{P}^{*}=\left(p_{x}^{*}\right)_{x \in \mathcal{X}}$ is obtained by solving the following feasibility problem [17, Section 4.1]

$$
\begin{array}{ll}
\text { Find } & \mathbf{P}^{*}=\left(p_{x}^{*}\right)_{x \in \mathcal{X}} \\
\text { subject to } & 0 \leq p_{x}^{*} \leq 1 \quad, x=1,2, \ldots, X \\
& \sum_{x \in \mathcal{X}_{j}} p_{x}^{*}=b_{j}^{*} \quad, j=1,2, \ldots, J \\
& \sum_{x=1}^{X} p_{x}^{*}=1
\end{array}
$$

\section{Simulation \& Numerical Results}

In each simulation run, two independent PPPs with intensities $\lambda_{b}$ and $\lambda_{u}$ are realized in $10 \times 10 \mathrm{~km}^{2}$. The SBSs independently cache the popular files according to the considered scenario, namely, Zipf or uniform. The user requests are realized using the Zipf distribution with parameter $\gamma$. Each users is associated to the nearest SBS that caches the requested file. The SBSs cater the files to the users in a multicast fashion using OSA. The hit probability is recored for the test user at the origin and the simulation is repeated 10000 times. Unless otherwise stated, network parameters are selected as follows; $\lambda_{b}=4 \mathrm{SBSs} / \mathrm{Km}^{2}, \lambda_{u}=40$ users $/ \mathrm{Km}^{2}, P=1$ watt, $\eta=4$.

Fig. 1a shows the hit probability versus the SINR threshold $\beta$. First, the matching between the analysis and simulations validates the developed mathematical model. The figure also shows that caching the files according to their popularity outperforms the uniform caching. However, the gain over the uniform caching is less at lower SINR threshold. This is because at lower SINR threshold value, the file can be catered via further SBSs. Hence, the significance of finding the file in a nearer SBS is less distinguishable. Also, the gain of popularity based caching is less for lower $\gamma$ as the the Zipf approaches the uniform distribution as $\gamma$ decreases.

Fig. 1b shows the hit probability versus the number of channels. First, the superiority of the optimal caching over the Zipf and uniform schemes is illuminated. Interestingly, the significance of the caching strategy on the hit probability 


$$
\begin{gathered}
\min ((n-2) M, k) \\
\left.\mathcal{P}_{n-1}\right|_{\infty}(\kappa)=\left.\sum_{t=M} \mathcal{P}_{n-2}\right|_{\infty}(t)\left(\begin{array}{c}
M \\
M-(\kappa-t)
\end{array}\right)\left(\frac{t}{|\mathbf{S}|}\right)^{M-(\kappa-t)}\left(1-\frac{t}{|\mathbf{S}|}\right)^{\kappa-t} \mathbb{1}_{\kappa-t \leq M}, \quad M \leq \kappa \leq \min ((n-1) M,|\mathbf{S}|), \\
\text { where }\left.\mathcal{P}_{1}\right|_{\infty}(\kappa)=\left\{\begin{array}{ll}
1, & \text { if } \kappa=M \\
0, & \text { otherwise. }
\end{array} \text { and }\left.\quad \mathcal{P}_{2}\right|_{\infty}(\kappa)=\left(\begin{array}{c}
M \\
2 M-\kappa
\end{array}\right)\left(\frac{M}{|\mathbf{S}|}\right)^{2 M-\kappa}\left(1-\frac{M}{|\mathbf{S}|}\right)^{\kappa-M}, M \leq \kappa \leq \min (2 M,|\mathbf{S}|) .\right.
\end{gathered}
$$

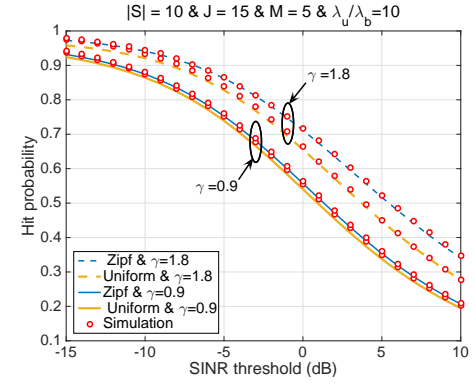

(a) $\mathcal{H}$ vs. $\beta \quad(M=5)$

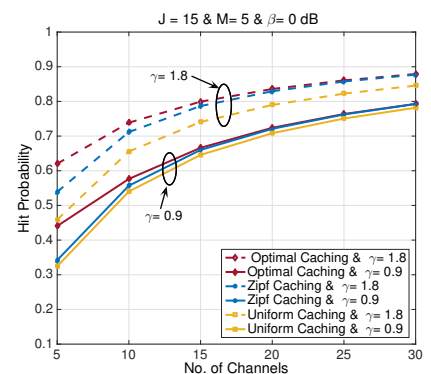

(b) $\mathcal{H}$ vs. $|\mathbf{S}|(M=10 \& \beta=0 \mathrm{~dB})$

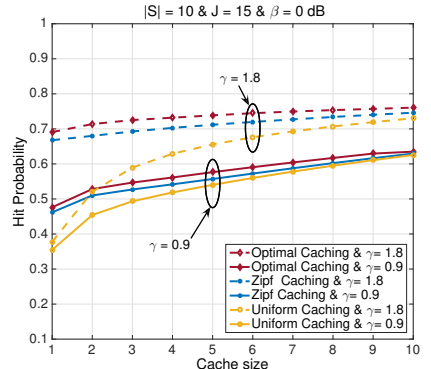

(c) $\mathcal{H}$ vs. $M(|\mathbf{S}|=10 \& \beta=0 \mathrm{~dB})$

Fig. 1: The hit probability versus SINR threshold, cache size and number of channels $\left(J=15 \& \frac{\lambda_{u}}{\lambda_{b}}=10\right)$

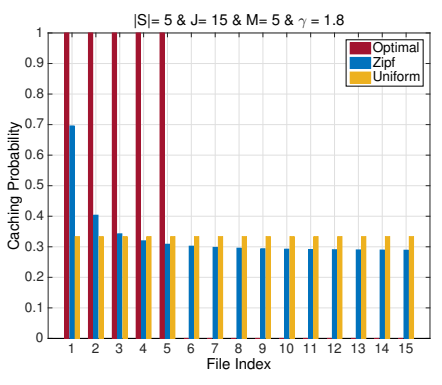

(a) $|\mathbf{S}|=5$

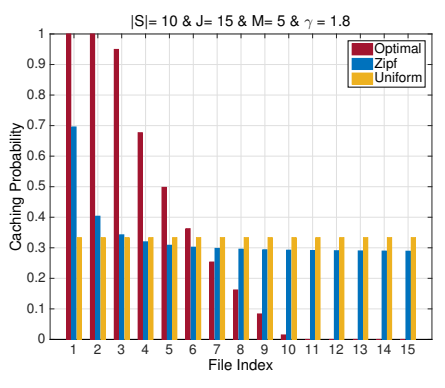

(b) $|\mathbf{S}|=10$

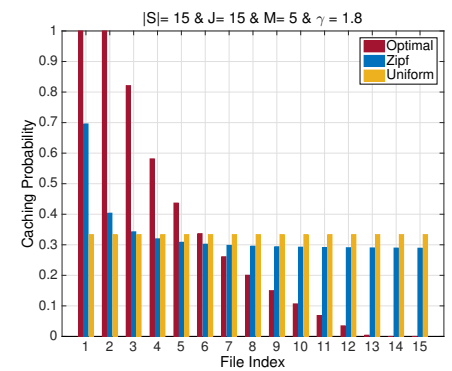

(c) $|\mathbf{S}|=15$

Fig. 2: The optimal caching $b_{j}^{*}$ versus the uniform and Zipf distributions $(J=15 \& M=5 \& \gamma=1.8)$

decreases when increasing the number of channels. Thanks to the OSA, the interference is relieved as the number of channel increases, which reduces the cost of fetching the file from a farther SBS. That is, increasing the number of channels makes farther SBSs capable to successfully cater for a file request, which decreases the significance of finding the file in a nearer SBS. Fig. 1c shows that the hit probability enhances when increasing the cache size, which is due to increasing the hit probability from a closer SBSs.

To better interpret the performance gains between the different caching strategies, we plot Fig. 2 to show the file caching probabilities in the buffer of a generic SBS. First, we note that the probabilities in Fig. 2 sum to $M$, which is the number of files that a SBS can store. It is obvious that the Zipf outperforms the uniform caching as it accounts for the file popularity. The optimal caching further adapts to the network conditions in addition to the file popularity, and hence, it outperforms the Zipf caching. For instance, Fig. 2a shows that when the interference conditions is too adverse (due to the small number of channels and/or high $\beta$ ), the optimal caching strategy caches only the most $M$ popular files in all SBSs.
Articulated differently, the optimal caching does not store any of the files $c_{j}$ for $M<j \leq J$ at any SBS. This is because any SBS farther than the closest one fails to cater a requested file with the required SINR threshold $\beta$. Consequently, the optimal caching strategy sacrifices the hitting probability of less popular files to guarantee that the most $M$ popular files are always served from the geographically closest SBS. Relieving the interference adversity (i.e., by increasing the number of channels or decreasing $\beta$ ), Figs. $2 \mathrm{~b}$ and $2 \mathrm{c}$ show that the optimal caching approaches the ZipF distribution and start to diversify the caching options over the SBSs according to the files popularity. The figure also shows that the number of sacrificed files that are never cached depends on the cost of fetching the files from farther SBSs.

\section{CONCLUSION}

This paper develops a mathematical framework, based on stochastic geometry, to characterize the hit probability of a cache-enabled multicast 5G network with SBS multi-channel capabilities and opportunistic spectrum access (OSA). Integral forms for the hit probability, are reduced to closed forms at practical special cases, are obtained. To this end, the 
optimal caching distribution that maximizes the hit probability is computed. The results signify the importance of OSA and optimal caching on the hit probability. In particular, at adverse interference conditions, the optimal caching significantly improves the hit probability when compared to the popularity or uniform based caching. When the number of channels increases, the OSA becomes effective and relieve the interference adversity. In this case, the cost to fetch the file from farther SBS decreases and the significance of the caching strategy becomes less distinguishable.

\section{APPENDIX A: PROOF OF LEMMA 1}

The PMF of the number of users request the file $c_{i}$ (i.e., belongs to the thinned PPP $\boldsymbol{\Phi}_{u, i}$ of intensity $a_{i} \lambda_{u}$ ) and are associated to a generic SBS that stores $c_{i}$ (i.e., belongs to the thinning PPP $\boldsymbol{\Phi}_{b, i}$ of intensity $b_{i} \lambda_{b}$ ), which is given by [18]

$$
\mathbb{P}\left[\mathcal{U}_{i}=k\right]=\frac{c^{c} \Gamma(k+c)\left(\frac{a_{i} \lambda_{u}}{b_{i} \lambda_{b}}\right)^{k}}{\Gamma(c) \Gamma(k+1)\left(\frac{a_{i} \lambda_{u}}{b_{i} \lambda_{b}}+c\right)^{k+c}}
$$

Under the multicast-transmission scheme, the probability that the file $c_{i}$ is sent by a generic SBS that caches it $\zeta_{i}$ can be expressed as

$$
\zeta_{i}=\mathbb{P}\left[\mathcal{U}_{i} \geq 1\right]=1-\mathbb{P}\left[\mathcal{U}_{i}=0\right]=1-\left[c^{-1} \frac{a_{i} \lambda_{u}}{b_{i} \lambda_{b}}+1\right]^{-c}
$$

Given that the SBS inside $r_{n}$ stores a combination $x \in \mathcal{X}_{-j}$, the conditional PMF of the number of used channels by this SBS is given by SBS stores

$$
\begin{aligned}
& \mathbb{P}\left[\mathcal{N}_{c,-j}=\kappa \mid \text { SBS stores a combination } x \in \mathcal{X}_{-j}\right] \\
& =\sum_{\{\mathcal{A} \subseteq x:|\mathcal{A}|=\kappa\}} \prod_{c_{i} \in \mathcal{A}} \zeta_{i} \prod_{c_{i} \in x \backslash \mathcal{A}}\left(1-\zeta_{i}\right), 0 \leq \kappa \leq M
\end{aligned}
$$

knowing that the SBS inside $r_{n}$ stores a combination that contains $x \in \mathcal{X}_{-j}$ that does not contain the typical user's requested file $c_{j}$ by a probability $\frac{P_{x}}{\left(1-b_{j}\right)}$. Therefore, by averaging over all possible combinations in the set $\mathcal{X}_{-j}$, the unconditional PMF of $\mathcal{N}_{c,-j}$ in Lemma 1 is obtained.

\section{APPENDIX B: PROOF OF LEMMA 2}

Given that the number of used channels by a generic SBS to serve its associated users is $N_{c}=n$. The probability that the SBS randomly chooses a particular channel is given by

$$
\mathcal{T}_{\mid n}= \begin{cases}\frac{\left(\begin{array}{c}
|\mathbf{S}|-1 \\
n-1
\end{array}\right)}{\left(\begin{array}{c}
|\mathbf{S}| \\
n
\end{array}\right)}=\frac{n}{|\mathbf{S}|}, & \text { if } n \leq M<|\mathbf{S}| . \\
1, & \text { if }|\mathbf{S}| \leq n \leq M . \\
0, & \text { if } n>M .\end{cases}
$$

Therefore, the unconditional probability can be obtained as

$$
\begin{aligned}
\mathcal{T} & =\sum_{n=0}^{\infty} \mathcal{T}_{\mid n} \mathbb{P}\left[\mathcal{N}_{c}=n\right] \\
& =\left\{\begin{array}{l}
\left.\sum_{n=0}^{M} \mathbb{P} \mid \mathcal{N}_{c}=n\right] \frac{n}{|\mathbf{S}|} \quad, \text { if } M<|\mathbf{S}| . \\
\sum_{n=0}^{M} \mathbb{P}\left[\mathcal{N}_{c}=n\right] \frac{n}{|\mathbf{S}|}+\sum_{n=|\mathbf{S}|}^{M} \mathbb{P}\left[\mathcal{N}_{c}=n\right] \\
=1-\sum_{n=0}^{|\mathbf{S}|-1} \frac{|\mathbf{S}|-n}{|\mathbf{S}|} \mathbb{P}\left[\mathcal{N}_{c}=n\right] \quad, \text { if } M \geq|\mathbf{S}| .
\end{array}\right.
\end{aligned}
$$

The last equality comes from the fact that $\sum_{n=0}^{\infty} \mathbb{P}\left[\mathcal{N}_{c}=\right.$ $n]=1$, thus $\sum_{n=|\mathbf{S}|}^{M} \mathbb{P}\left[\mathcal{N}_{c}=n\right]=1-\sum_{n=0}^{|\mathbf{S}|-1} \mathbb{P}\left[\mathcal{N}_{c}=n\right]$.

\section{REFERENCES}

[1] V. Cisco, "Forecast and methodology, 2015-2020."

[2] E. Bastug, M. Bennis, and M. Debbah, "Living on the edge: The role of proactive caching in $5 \mathrm{~g}$ wireless networks," IEEE Communications Magazine, vol. 52, no. 8, pp. 82-89, 2014.

[3] K. Shanmugam, N. Golrezaei, A. G. Dimakis, A. F. Molisch, and G. Caire, "Femtocaching: Wireless content delivery through distributed caching helpers," IEEE Transactions on Information Theory, vol. 59, no. 12 , pp. 8402-8413, 2013.

[4] K. Poularakis, G. Iosifidis, and L. Tassiulas, "Approximation algorithms for mobile data caching in small cell networks," IEEE Transactions on Communications, vol. 62, no. 10, pp. 3665-3677, 2014.

[5] B. Blaszczyszyn and A. Giovanidis, "Optimal geographic caching in cellular networks," in 2015 IEEE International Conference on Communications (ICC). IEEE, 2015, pp. 3358-3363.

[6] B. Serbetci and J. Goseling, "On optimal geographical caching in heterogeneous cellular networks," arXiv preprint arXiv:1601.07322, 2016.

[7] M. Afshang and H. S. Dhillon, "Optimal geographic caching in finite wireless networks," in Signal Processing Advances in Wireless Communications (SPAWC), 2016 IEEE 17th International Workshop on. IEEE, 2016, pp. $1-5$.

[8] Y. Cui, Y. Wu, and D. Jiang, "Analysis and optimization of caching and multicasting in large-scale cache-enabled information-centric networks," in 2015 IEEE Global Communications Conference (GLOBECOM). IEEE, 2015, pp. 1-7.

[9] A. H. Sakr and E. Hossain, "Cognitive and energy harvesting-based d2d communication in cellular networks: Stochastic geometry modeling and analysis," IEEE Transactions on Communications, vol. 63, no. 5, pp. 1867-1880, 2015.

[10] M. Emara, H. ElSawy, S. Sorour, S. Al-Ghadhban, M.-S. Alouini, and T. Y. Al-Naffouri, "Stochastic geometry model for multi-channel fog radio access networks," in Modeling \& Optimization in Mobile, Ad Hoc \& Wireless Networks (WiOpt), 2017 15th International Symposium on. IEEE, 2017, pp. 1-6.

[11] S. N. Chiu, D. Stoyan, W. S. Kendall, and J. Mecke, Stochastic geometry and its applications. John Wiley \& Sons, 2013.

[12] H. ElSawy and E. Hossain, "Channel assignment and opportunistic spectrum access in two-tier cellular networks with cognitive small cells," in 2013 IEEE Global Communications Conference (GLOBECOM). IEEE, 2013, pp. 4477-4482.

[13] N. Lee, D. Morales-Jimenez, A. Lozano, and R. W. Heath, "Spectral efficiency of dynamic coordinated beamforming: A stochastic geometry approach," IEEE Transactions on Wireless Communications, vol. 14, no. 1, pp. 230-241, 2015.

[14] R. Arshad, H. ElSawy, S. Sorour, T. Y. Al-Naffouri, and M.-S. Alouini, "Velocity-aware handover management in two-tier cellular networks," IEEE Transactions on Wireless Communications, vol. 16, no. 3, pp. $1851-1867,2017$.

[15] I. S. Gradshteyn and I. M. Ryzhik, Table of Integrals, Series, and Products, Seventh Edition. Academic Press, 2007.

[16] Y. Cui, D. Jiang, and Y. Wu, "Analysis and optimization of caching and multicasting in large-scale cache-enabled wireless networks," IEEE Transactions on Wireless Communications, vol. 15, no. 7, pp. 51015112, 2016.

[17] S. Boyd and L. Vandenberghe, Convex optimization. Cambridge university press, 2004.

[18] H. ElSawy and E. Hossain, "Two-tier hetnets with cognitive femtocells: Downlink performance modeling and analysis in a multichannel environment," IEEE Transactions on Mobile Computing, vol. 13, no. 3, pp. 649-663, 2014. 